\title{
Thiourea dioxide: An efficient and reusable organocatalyst for the rapid one-pot synthesis of pyrano[4,3-b]pyran derivatives in water
}

\author{
Majid Ghashang a, Syed Sheik Mansoor b,*, Krishnamoorthy Aswin b \\ a Faculty of Sciences, Najafabad Branch, Islamic Azad University, Najafabad, Esfahan, Iran \\ ${ }^{\mathrm{b}}$ Research Department of Chemistry, Bioactive Organic Molecule Synthetic Unit, C. Abdul Hakeem College, Melvisharam - 632 509, Tamil Nadu, India
}

\section{A R T I C L E I N F O}

\section{Article history:}

Received 9 August 2013

Accepted 9 October 2013

Published 20 January 2014

\section{Keywords:}

Pyrano[4,3-b]pyran

Thiourea dioxide

4-Hydroxy-6-methylpyran-2-one

One-pot synthesis

\begin{abstract}
A B S T R A C T
A series of pyrano[4,3- $b$ ]pyran derivatives have been synthesized in excellent yields by the reaction of aromatic aldehydes with malononitrile or cyanoacetate and 4-hydroxy-6-methylpyran-2-one in water at $80^{\circ} \mathrm{C}$, with the transformation being catalyzed by an aqueous solution of thiourea dioxide (TUD). Upon completion of the reaction, the product was isolated by filtration or extraction and the remaining aqueous TUD solution could be reused several times without any discernible impact on its catalytic activity. This procedure offers several advantages over existing procedures, including high yields, operational simplicity, the use of a non-toxic catalyst and solvent, short reaction time and minimum pollution of the environment, making it a useful and attractive process for the preparation of pyrano[4,3-b]pyran derivatives.
\end{abstract}

(C) 2014, Dalian Institute of Chemical Physics, Chinese Academy of Sciences. Published by Elsevier B.V. All rights reserved.

\section{Introduction}

Multi-component reactions (MCRs) represent an attractive synthetic strategy for rapid and efficient generation of compound library because they allow for high levels of diversity to be readily achieved by simply varying the reacting components. MCRs have attracted considerable attention from synthetic organic chemists for the construction of highly functionalized organic molecules and pharmacologically important heterocyclic compounds [1-3].

The development of eco-friendly methodologies that are environmentally benign and waste-free, as well as being able to produce the desired products in high purity, have received significant levels of attention in recent years because of the increasing tendency of the chemical industry towards greener processes. To reach this goal of greener processes, the use of green reaction media, reagents, and catalysts has been investigated extensively. Water represents a unique solvent in organic synthesis, because it is not only abundant, inexpensive and environmentally benign, but also shows novel reactivity and selectivity for the synthesis of simple organic compounds such as pharmaceutical products, agrochemicals, fine chemicals, and synthetic intermediates [4].

The development of new methodologies for the synthesis of pyrans continues to be a subject of considerable interest to synthetic and medicinal chemists. Pyrans are important oxygenated heterocyclic compounds endowed with a wide range of biological activity, including antiviral [5], mutagenic [6], antimicrobial [7], antiproliferative [8], sex pheromone [9], antitumor [10], and antitubercular and antimicrobial [11] activity. The development of novel methodologies for the synthesis of pyran derivatives has therefore attracted significant levels of interest.

In recent years, several new and efficient methods have been developed for the synthesis of pyran derivatives with the aim of providing access to heterocyclic systems exhibiting high

\footnotetext{
* Corresponding author. Tel/Fax: +91-04172-266487; E-mail: smansoors2000@yahoo.co.in

This work was supported by Research Department of Chemistry, C. Abdul Hakeem College, Melvisharam - 632 509, Tamil Nadu, India. DOI: 10.1016/S1872-2067(12)60727-X | http://www.sciencedirect.com/science/journal/18722067 | Chin. J. Catal., Vol. 35, No. 1, January 2014
} 
levels of biologically activity using a range of different catalysts, such as potassium sodium tartrate [12], magnetic nanoparticle supported hyperbranched polyglycerol [13], tetrabutylammonium bromide (TBAB) [14], baker's yeast [15], amino functionalized ionic liquid [16] and SB-DABCO [17]. In contrast, far fewer methods have been reported for the synthesis of pyrano[4,3- $b]$ pyran derivatives via the one-pot reaction of aromatic aldehydes with malononitrile and 4-hydroxy-6-methyl-pyran2-one using catalysts such as $\mathrm{KF} / \mathrm{Al}_{2} \mathrm{O}_{3}$ [18], alum [KAl(SO $\left.)_{4}\right)_{2} \cdot 12 \mathrm{H}_{2} \mathrm{O}$ ] [19], mesoporous $\mathrm{NH}_{2}-\mathrm{MCM}-41$ [20], and ammonium acetate under solvent-free conditions using the grinding method [21]. Most of these procedures are conducted under reflux conditions in organic solvents over extended reaction periods, and require the use of expensive catalysts as well as involving tedious work-up procedures. With these points in mind, we decided to investigate the application of thiourea dioxide (TUD) as an organocatalyst for the synthesis of 2-amino-7-methyl-5-oxo-4-phenyl-4H,5 $H$-pyrano[4,3- $b$ ]pyran3 -carbonitrile derivatives in water.

TUD has recently received considerable attention as an efficient catalyst for the construction of carbon-carbon and carbon hetero atom bonds [22-24] because of its eco-friendly nature, easy handling, high reactivity and facile work-up procedures. The use of TUD as a novel organocatalyst has been reported on several occasions, including the one-pot synthesis of a library of novel heterocyclic compounds [22], the hydrolysis of imines [23] and the synthesis of naphthopyran derivatives [24].

TUD can be readily prepared [25] by the oxidation of thiourea with hydrogen peroxide and is a highly stable material. TUD possesses the ability to activate organic substrates through hydrogen bonding. Furthermore, TUD can form strong hydrogen bonding interaction and provide higher levels of activation than the corresponding thiourea because it possesses two extra oxygen atoms. In terms of its physical properties, TUD is insoluble in most common organic solvents and can therefore be readily recovered at the end of the reaction and recycled. Among the most recent advancements in organic and synthetic chemistry, the discovery of catalysis by small organic molecules referred to as "organocatalysts" represents a significant breakthrough, which offers a series of significant advantages, including low activation energy, high stability, a metal-free environment, and mild reaction conditions [22]. Furthermore, these processes are generally robust, inexpensive, readily available, and non-toxic. These processes are generally tolerant towards moisture and oxygen, and demanding reaction conditions, such as the requirement for an inert atmosphere, low temperatures, or absolute solvents, are in many instances not required. Organocatalytic methods seem to be especially attractive for the preparation of drug-like compounds because of the absence of transition metals [4], which can contaminate the products.

During the course of our recent studies towards improving the eco-compatibility of certain organic processes [26-29], we became particularly interested in the synthesis of a series of potentially active pyrano[4,3-b]pyran derivatives in water to develop environmentally benign reactions. Herein, we wish to report the development of an efficient process for the preparation of pyrano[4,3-b]pyran derivatives via the one-pot reaction

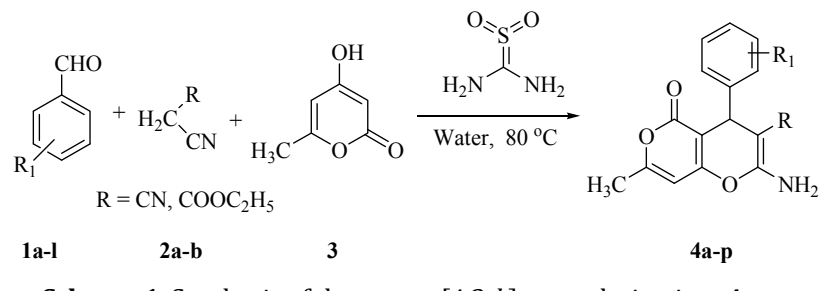

Scheme 1. Synthesis of the pyrano[4,3-b]pyran derivatives 4a-p.

of aromatic aldehydes, malononitrile or cyanoacetate and 4-hydroxy-6-methylpyran-2-one, with the process being catalyzed by TUD in water (Scheme 1).

\section{Experimental}

\subsection{Apparatus and analysis}

All of the chemicals used in the current study were purchased from Merck, Fluka and Sigma-Aldrich. Thiourea and hydrogen peroxide were used for the preparation of TUD. A variety of different substituted benzaldehydes were used in the current study, including the bromo, chloro, fluoro, nitro, methyl and methoxy substituted systems. Malononitrile or cyanoacetate and 4-hydroxy-6-methylpyran-2-one were also used for the synthesis. Several solvents, including 1,4-dioxane, acetonitrile, THF, $\mathrm{CH}_{2} \mathrm{Cl}_{2}, \mathrm{CHCl}_{3}$, methanol, ethanol and water, were used to study the optimization of the solvent for the synthesis of 2-amino-7-methyl-5-oxo-4-(4-bromophenyl)-4H,5 $H$-pyrano $[4,3-b]$ pyran-3-carbonitrile. All of the quoted yields refer to the yields of the isolated products unless otherwise stated. ${ }^{1} \mathrm{H}-\mathrm{NMR}$ $(500 \mathrm{MHz})$ and ${ }^{13} \mathrm{C}-\mathrm{NMR}(125 \mathrm{MHz})$ spectra were obtained using a Bruker DRX-500 Avance spectrometer (Bruker) at ambient temperature, using TMS as internal reference standard. FT-IR spectra were obtained as $\mathrm{KBr}$ discs on a Shimadzu spectrometer (Shimadzu). Mass spectra were determined on a Varion - Saturn 2000 GC/MS instrument (Varian). Elemental analyses were measured using a Perkin Elmer 2400 CHN elemental analyzer flowchart (Perkin Elmer).

\subsection{General procedure for the synthesis of the pyrano[4,3-b]pyran derivatives (4a-p)}

A dry 50-ml flask was charged with the aromatic aldehyde $\mathbf{1}$ (1.0 mmol), malononitrile or cyanoacetate 2 (1.2 mmol), 4-hydroxy-6-methylpyran-2-one 3 (1 mmol), TUD (molar fraction $(x) 10 \%)$ and water $(5 \mathrm{ml})$, and the resulting mixture was stirred at $80{ }^{\circ} \mathrm{C}$ for $30-60 \mathrm{~min}$. The mixture was then poured into water $(100 \mathrm{ml})$, and the resulting solid was removed by filtration, and washed with water. The remaining aqueous thiourea dioxide was collected and reused without any further processing for subsequent runs. The reaction products were identified by comparing their physical and spectral data (i.e., IR, ${ }^{1} \mathrm{H}$ and ${ }^{13} \mathrm{C}$ NMR and MS) with those reported in the literature for the same compounds. The crude products were purified by recrystallization from DMF and water to give 4a-p.

\subsection{Spectral data for the synthesized compounds (4a-p)}


2-Amino-7-methyl-5-oxo-4-(4-bromophenyl)-4H,5H-pyrano [4,3-b]pyran-3-carbonitrile (4a). Pale yellow crystals; m.p.: 222-224 ${ }^{\circ} \mathrm{C}$; IR (KBr, cm-1): 3377 and $3307\left(\mathrm{NH}_{2}\right), 3166(\mathrm{ArH})$, 2222 (CN), 1706 (C=0); ${ }^{1} \mathrm{H}$ NMR (500 MHz, DMSO-d 6 ) $\delta: 2.23$ (s, $\left.3 \mathrm{H}, \mathrm{CH}_{3}\right), 4.42(\mathrm{~s}, 1 \mathrm{H}, \mathrm{CH}), 6.52(\mathrm{~s}, 1 \mathrm{H},=\mathrm{CH}), 6.77\left(\mathrm{~s}, 2 \mathrm{H}, \mathrm{NH}_{2}\right)$, $7.17(\mathrm{~d}, 2 \mathrm{H}, J=8.2 \mathrm{~Hz}, \mathrm{Ar}-\mathrm{H}), 7.44(\mathrm{~d}, 2 \mathrm{H}, J=8.2 \mathrm{~Hz}, \mathrm{Ar}-\mathrm{H}) \mathrm{ppm}$; ${ }^{13} \mathrm{C}$ NMR $\left(125 \mathrm{MHz}\right.$, DMSO- $\left.d_{6}\right) \delta: 22.6,26.9,57.4,101.0,102.8$, 118.0, 125.8, 128.2, 129.7, 137.7, 144.4, 160.5, 164.8, 176.8 ppm; MS(ESI): $M r / z \quad 359.9 \quad[\mathrm{M}+\mathrm{H}]^{+}$; Anal. Calcd for $\mathrm{C}_{16} \mathrm{H}_{11} \mathrm{BrN}_{2} \mathrm{O}_{3}$ : C 53.50\%, H 3.06\%, N 7.80\%; Found: C 53.42\%, H $3.02 \%$, N 7.77\%.

2-Amino-7-methyl-5-oxo-4-(3-nitrophenyl)-4H,5H-pyrano [4,3-b]pyran-3-carbonitrile (4b). Pale yellow crystals; m.p.: 236-238 ${ }^{\circ} \mathrm{C}$; IR (KBr, cm-1): 3383 and $3313\left(\mathrm{NH}_{2}\right), 3172(\mathrm{ArH})$, $2214(\mathrm{CN}), 1711$ (C=0); ${ }^{1} \mathrm{H}$ NMR (500 MHz, DMSO-d 6 ) $\delta: 2.14$ (s, $\left.3 \mathrm{H}, \mathrm{CH}_{3}\right), 4.38(\mathrm{~s}, 1 \mathrm{H}, \mathrm{CH}), 6.40(\mathrm{~s}, 1 \mathrm{H}, \mathrm{C}=\mathrm{CH}), 6.84\left(\mathrm{~s}, 2 \mathrm{H}, \mathrm{NH}_{2}\right)$, $7.42(\mathrm{t}, J=8.0 \mathrm{~Hz}, 1 \mathrm{H}, \mathrm{ArH}), 7.63\left(\mathrm{tt}, J=8.2 \mathrm{~Hz}, J^{\prime}=1.3 \mathrm{~Hz}, 1 \mathrm{H}\right.$, Ar-H), $7.82(\mathrm{t}, J=2.2 \mathrm{~Hz}, 1 \mathrm{H}, \mathrm{Ar}-\mathrm{H}), 8.03\left(\mathrm{dd}, \mathrm{dd}, J=8.2 \mathrm{~Hz}, J^{\prime}=\right.$ $\left.2.2 \mathrm{~Hz}, J^{\prime \prime}=1.3 \mathrm{~Hz}, 1 \mathrm{H}, \mathrm{A}-\mathrm{rH}\right) \mathrm{ppm} ;{ }^{13} \mathrm{C}$ NMR (125 MHz, DMSO- $\left.d_{6}\right) \delta: 23.3,26.7,56.9,100.7,103.3,117.7,126.3,128.6$, $129.5,138.2,143.7,161.3,165.2,177.2 \mathrm{ppm}$; MS(ESI): $M r / z$ $326[\mathrm{M}+\mathrm{H}]^{+}$; Anal. Calcd for $\mathrm{C}_{16} \mathrm{H}_{11} \mathrm{~N}_{3} \mathrm{O}_{5}$ : C 59.08\%, H 3.38\%, N 12.92\%; Found: C 59.06\%, H 3.39\%, N $12.86 \%$.

2-Amino-7-methyl-5-oxo-4-(4-chlorophenyl)-4H,5 H-pyrano [4,3-b]pyran-3-carbonitrile (4c). Pale yellow crystals; m.p.: 231-233 ${ }^{\circ} \mathrm{C}$; IR (KBr, cm-1): 3375 and $3318\left(\mathrm{NH}_{2}\right), 3177(\mathrm{ArH})$, $2224(\mathrm{CN}), 1716$ (C=0); ${ }^{1} \mathrm{H}$ NMR (500 MHz, DMSO-d 6 ) $\delta: 2.11$ (s, $3 \mathrm{H}, \mathrm{CH} 3), 4.53(\mathrm{~s}, 1 \mathrm{H}, \mathrm{CH}), 6.47$ (s, 1H, C=CH), $6.68\left(\mathrm{~s}, 2 \mathrm{H}, \mathrm{NH}_{2}\right.$ ), $7.13(\mathrm{~d}, 2 \mathrm{H}, J=8.2 \mathrm{~Hz}, \mathrm{ArH}), 7.53(\mathrm{~d}, 2 \mathrm{H}, \quad J=8.2 \mathrm{~Hz}, \mathrm{ArH}) \mathrm{ppm}$; ${ }^{13} \mathrm{C}$ NMR (125 MHz, DMSO-d6) $\delta: 23.1,27.3,56.7,101.4,103.0$, 118.3, 126.0, 129.0, 130.3, 138.0, 143.9, 160.9, 164.9, 177.1 ppm; MS(ESI): $M r / z 315$ [M+H]+; Anal. Calcd for $\mathrm{C}_{16} \mathrm{H}_{11} \mathrm{ClN}_{2} \mathrm{O}_{3}$ : C $61.06 \%$, H $3.50 \%$, N 8.90\%; Found: C $60.96 \%$, H 3.44\%, N $8.85 \%$.

2-Amino-7-methyl-5-oxo-4-(3-chlorophenyl)-4H,5H-pyrano [4,3-b]pyran-3-carbonitrile (4d). Pale yellow crystals; m.p.: 254-256 ${ }^{\circ} \mathrm{C}$; IR (KBr, cm$\left.{ }^{-1}\right)$ : 3386 and $3309\left(\mathrm{NH}_{2}\right), 3174(\mathrm{ArH})$, $2211(\mathrm{CN}), 1701(\mathrm{C}=0)$ ); ${ }^{1} \mathrm{H}$ NMR (500 MHz, DMSO- $\left.d_{6}\right) \delta: 2.22$ (s, $\left.3 \mathrm{H}, \mathrm{CH}_{3}\right), 4.39(\mathrm{~s}, 1 \mathrm{H}, \mathrm{CH}), 6.39$ (s, 1H, C=CH), $6.73\left(\mathrm{~s}, 2 \mathrm{H}, \mathrm{NH}_{2}\right)$, 7.33-7.53 (m, 4H, Ar-H) ppm; ${ }^{13} \mathrm{C}$ NMR $\left(125 \mathrm{MHz}\right.$, DMSO- $\left.d_{6}\right) \delta$ : 23.6, 27.0, 56.5, 100.9, 102.7, 117.9, 125.5, 129.1, 130.0, 137.8, 144.3, 161.4, 165.4, 176.9 ppm; MS(ESI): $M r / z ~ 315[\mathrm{M}+\mathrm{H}]^{+}$; Anal. Calcd for $\mathrm{C}_{16} \mathrm{H}_{11} \mathrm{ClN}_{2} \mathrm{O}_{3}$ : C $61.06 \%$, H 3.50\%, N 8.90\%; Found: C $61.01 \%$, H 3.51\%, N 8.83\%.

2-Amino-7-methyl-5-oxo-4-(2-chlorophenyl)-4H,5H-pyrano [4,3-b]pyran-3-carbonitrile (4e). Pale yellow crystals; m.p.: 270-271 ${ }^{\circ} \mathrm{C}$; IR $\left(\mathrm{KBr}, \mathrm{cm}^{-1}\right)$ : 3385 and $3311\left(\mathrm{NH}_{2}\right), 3169(\mathrm{ArH})$, 2208 (CN), 1717 (C=0); ${ }^{1} \mathrm{H}$ NMR (500 MHz, DMSO-d 6 ) $\delta: 2.09$ (s, $\left.3 \mathrm{H}, \mathrm{CH}_{3}\right), 4.41(\mathrm{~s}, 1 \mathrm{H}, \mathrm{CH}), 6.51(\mathrm{~s}, 1 \mathrm{H}, \mathrm{C}=\mathrm{CH}), 6.82\left(\mathrm{~s}, 2 \mathrm{H}, \mathrm{NH}_{2}\right)$, 7.22-7.48 (m, 4H, Ar-H) ppm; ${ }^{13} \mathrm{C}$ NMR (125 MHz, DMSO- $\left.d_{6}\right) \delta$ : 22.9, 27.4, 57.0, 101.1, 103.4, 118.4, 126.1, 128.8, 129.6, 137.6, 143.6, 160.8, 164.7, 177.0 ppm; MS(ESI): Mr/z $315[\mathrm{M}+\mathrm{H}]^{+}$; Anal. Calcd for $\mathrm{C}_{16} \mathrm{H}_{11} \mathrm{ClN}_{2} \mathrm{O}_{3}$ : C $61.06 \%$, H 3.50\%, N 8.90\%; Found: C 60.99\%, H 3.48\%, N 8.81\%.

2-Amino-7-methyl-5-oxo-4-(3,4-dichlorophenyl)-4H,5 Hpyrano[4,3-b]pyran-3-carbonitrile (4f). Pale yellow crystals; m.p.: $238-240{ }^{\circ} \mathrm{C}$; IR $\left(\mathrm{KBr}, \mathrm{cm}^{-1}\right)$ : 3382 and $3312\left(\mathrm{NH}_{2}\right), 3167$
(ArH), 2216 (CN), 1709 (C=0); ${ }^{1} \mathrm{H}$ NMR (500 MHz, DMSO-d $) \delta$ : $2.13\left(\mathrm{~s}, 3 \mathrm{H}, \mathrm{CH}_{3}\right), 4.54(\mathrm{~s}, 1 \mathrm{H}, \mathrm{CH}), 6.48(\mathrm{~s}, 1 \mathrm{H}, \mathrm{C}=\mathrm{CH}), 6.75(\mathrm{~s}$, $2 \mathrm{H}, \mathrm{NH}_{2}$ ), 7.19 (dd, $\left.1 \mathrm{H}, J=8.0 \mathrm{~Hz}, J^{\prime}=2.0 \mathrm{~Hz}, \mathrm{ArH}\right), 7.44$ (d, $1 \mathrm{H}, J$ $=2.0 \mathrm{~Hz}, \mathrm{ArH}), 7.66$ (d, 1H, $J=8.0 \mathrm{~Hz}, \mathrm{ArH}) \mathrm{ppm} ;{ }^{13} \mathrm{C}$ NMR $(125$ MHz, DMSO-d $) \delta: 23.4,26.9,56.4,101.4,102.6,117.8,125.9$, 128.5, 129.7, 138.5, 144.0, 161.5, 165.5, 176.6 ppm; MS(ESI): $\mathrm{Mr} / \mathrm{z} 349.5$ [M+H]+; Anal. Calcd for $\mathrm{C}_{16} \mathrm{H}_{10} \mathrm{Cl}_{2} \mathrm{~N}_{2} \mathrm{O}_{3}$ : C $55.03 \%, \mathrm{H}$ 2.87\%, N 8.03\%; Found: C 54.91\%, H 2.82\%, N 8.00\%.

2-Amino-7-methyl-5-oxo-4-(2,4-dichlorophenyl)- $4 \mathrm{H}, 5 \mathrm{H}$ pyrano[4,3-b]pyran-3-carbonitrile (4g). Pale yellow crystals; m.p.: 232-234 ${ }^{\circ} \mathrm{C}$; IR $\left(\mathrm{KBr}, \mathrm{cm}^{-1}\right)$ : 3380 and $3316\left(\mathrm{NH}_{2}\right), 3178$ (ArH), 2210 (CN), 1713 (C=0); ${ }^{1} \mathrm{H}$ NMR (500 MHz, DMSO-d6) $\delta$ : $2.17\left(\mathrm{~s}, 3 \mathrm{H}, \mathrm{CH}_{3}\right), 4.39(\mathrm{~s}, 1 \mathrm{H}, \mathrm{CH}), 6.41(\mathrm{~s}, 1 \mathrm{H}, \mathrm{C}=\mathrm{CH}), 6.81(\mathrm{~s}, 2 \mathrm{H}$, $\left.\mathrm{NH}_{2}\right), 7.25(\mathrm{~d}, 1 \mathrm{H}, J=8.0 \mathrm{~Hz}, \mathrm{Ar}-\mathrm{H}), 7.36\left(\mathrm{dd}, 1 \mathrm{H}, J=8.0 \mathrm{~Hz}, J^{\prime}=\right.$ $2.0 \mathrm{~Hz}, \mathrm{Ar}-\mathrm{H}$ ), 7.55 (d, 1H, J = 2.0 Hz, Ar-H) ppm; ${ }^{13} \mathrm{C}$ NMR (125 MHz, DMSO-d6) $\delta: 22.5,26.6,57.3,100.7,102.9,117.5,125.4$, 129.3, 130.1, 137.9, 144.5, 160.6, 164.6, 177.4 ppm; MS(ESI): $\mathrm{Mr} / \mathrm{z} 349.7$ [M+H] +; Anal. Calcd for $\mathrm{C}_{16} \mathrm{H}_{10} \mathrm{Cl}_{2} \mathrm{~N}_{2} \mathrm{O}_{3}$ : C $55.03 \%, \mathrm{H}$ 2.87\%, N 8.03\%; Found: C 55.01\%, H 2.84\%, N 8.06\%.

2-Amino-7-methyl-5-oxo-4-(4-chloro-2-nitrophenyl)-4H,5H -pyrano[4,3- $b]$ pyran-3-carbonitrile (4h). Pale yellow crystals; m.p.: $246-248^{\circ} \mathrm{C}$; IR $\left(\mathrm{KBr}, \mathrm{cm}^{-1}\right): 3389$ and $3309\left(\mathrm{NH}_{2}\right), 3169$ (ArH), 2220 (CN), 1701 (C=0); ${ }^{1} \mathrm{H}$ NMR (500 MHz, DMSO-d $) \delta$ : $2.21\left(\mathrm{~s}, 3 \mathrm{H}, \mathrm{CH}_{3}\right), 4.54(\mathrm{~s}, 1 \mathrm{H}, \mathrm{CH}), 6.37(\mathrm{~s}, 1 \mathrm{H}, \mathrm{C}=\mathrm{CH}), 6.74(\mathrm{~s}$, $\left.2 \mathrm{H}, \mathrm{NH}_{2}\right), 7.47(\mathrm{~d}, 1 \mathrm{H}, J=2.0 \mathrm{~Hz}, \mathrm{Ar}-\mathrm{H}), 7.55\left(\mathrm{dd}, 1 \mathrm{H}, J=8.6 \mathrm{~Hz}, J^{\prime}\right.$ $=2.0 \mathrm{~Hz}, \mathrm{Ar}-\mathrm{H}), 7.88$ (d, $1 \mathrm{H}, J=8.6 \mathrm{~Hz}, \mathrm{Ar}-\mathrm{H}) \mathrm{ppm} ;{ }^{13} \mathrm{C} \mathrm{NMR}$ $\left(125 \mathrm{MHz}, \mathrm{DMSO}-d_{6}\right) \delta: 23.0,27.0,56.8,100.9,103.1,118.6$, 126.2, 129.2, 130.5, 138.4, 143.9, 160.9, 165.0, 176.5 ppm; MS(ESI): $M r / z 360(\mathrm{M}+\mathrm{H})^{+}$; Anal. Calcd for $\mathrm{C}_{16} \mathrm{H}_{10} \mathrm{ClN}_{3} \mathrm{O}_{5}$ : C $53.42 \%$, H 2.78\%, N 11.68\%; Found: C 53.33\%, H 2.74\%, N $11.69 \%$.

2-Amino-7-methyl-5-oxo-4-(4-methylphenyl)-4H,5H-pyrano [4,3-b]pyran-3-carbonitrile (4i). Pale yellow crystals; m.p.: 216-218 ${ }^{\circ} \mathrm{C}$; IR $\left(\mathrm{KBr}, \mathrm{cm}^{-1}\right)$ : 3362 and $3301\left(\mathrm{NH}_{2}\right), 3159(\mathrm{ArH})$, $2223(\mathrm{CN}), 1709$ (C=0); ${ }^{1} \mathrm{H}$ NMR (500 MHz, DMSO-d $) \delta: 2.15$ (s, $\left.3 \mathrm{H}, \mathrm{CH}_{3}\right), 2.25\left(\mathrm{~s}, 3 \mathrm{H}, \mathrm{CH}_{3}\right), 4.49(\mathrm{~s}, 1 \mathrm{H}, \mathrm{CH}), 6.47(\mathrm{~s}, 1 \mathrm{H}, \mathrm{C}=\mathrm{CH})$, 6.76 (s, 2H, NH $\left.\mathrm{N}_{2}\right), 7.19$ (d, 2H, $\left.J=8.2 \mathrm{~Hz}, \mathrm{Ar}-\mathrm{H}\right), 7.48$ (d, 2H, $J=$ $8.2 \mathrm{~Hz}, \mathrm{Ar}-\mathrm{H}$ ) ppm; ${ }^{13} \mathrm{C}$ NMR (125 MHz, DMSO-d $) \delta: 20.7,22.4$, 26.5, 57.1, 100.8, 102.4, 117.8, 125.3, 128.4, 129.6, 137.7, 144.1, 160.2, 164.4, 177.0 ppm; MS(ESI): $M r / z 295[\mathrm{M}+\mathrm{H}]^{+}$; Anal. Calcd for $\mathrm{C}_{17} \mathrm{H}_{14} \mathrm{~N}_{2} \mathrm{O}_{3}$ : C $69.38 \%, \mathrm{H} 4.76 \%$, N 9.52\%; Found: $\mathrm{C}$ $69.33 \%, \mathrm{H} 4.72 \%$, N 9.47\%.

2-Amino-7-methyl-5-oxo-4-(4-methoxyphenyl)- $4 \mathrm{H}, 5 \mathrm{H}$ pyrano[4,3-b]pyran-3-carbonitrile (4j). Pale yellow crystals; m.p.: $228-230{ }^{\circ} \mathrm{C}$; IR $\left(\mathrm{KBr}, \mathrm{cm}^{-1}\right): 3370$ and $3311\left(\mathrm{NH}_{2}\right), 3163$ (ArH), 2217 (CN), 1713 (C=0); ${ }^{1} \mathrm{H}$ NMR (500 MHz, DMSO-d $) \delta$ : $2.21\left(\mathrm{~s}, 3 \mathrm{H}, \mathrm{CH}_{3}\right), 3.62\left(\mathrm{~s}, 3 \mathrm{H}, \mathrm{OCH}_{3}\right), 4.43(\mathrm{~s}, 1 \mathrm{H}, \mathrm{CH}), 6.54(\mathrm{~s}$, $1 \mathrm{H}, \mathrm{C}=\mathrm{CH}), 6.79$ (s, 2H, NH 2$), 7.10(\mathrm{~d}, 2 \mathrm{H}, J=8.2 \mathrm{~Hz}, \mathrm{Ar}-\mathrm{H}), 7.37$ (d, $2 \mathrm{H}, J=8.2 \mathrm{~Hz}, \mathrm{Ar}-\mathrm{H}) \mathrm{ppm} ;{ }^{13} \mathrm{C}$ NMR $(125 \mathrm{MHz}$, DMSO-d $) \delta$ : 22.3, 26.7, 54.1, 57.2, 101.2, 102.9, 118.0, 125.9, 128.0, 129.5, 137.2, 144.7, 161.0, 164.7, 176.7 ppm; MS(ESI): $M r / z ~ 311$ $[\mathrm{M}+\mathrm{H}]^{+}$; Anal. Calcd for $\mathrm{C}_{17} \mathrm{H}_{14} \mathrm{~N}_{2} \mathrm{O}_{4}$ : C $65.80 \%, \mathrm{H} 4.52 \%, \mathrm{~N}$ 9.03\%; Found: C 65.72\%, H 4.50\%, N 9.01\%.

Ethyl 2-amino-7-methyl-5-oxo-4-(4-fluorophenyl)-4 $\mathrm{H}, 5 \mathrm{H}$ pyrano[4,3-b]pyran-3-carboxylate (4k). Pale yellow crystals; m.p.: 202-204 ${ }^{\circ} \mathrm{C}$; IR $\left(\mathrm{KBr}, \mathrm{cm}^{-1}\right)$ : 3373 and $3311\left(\mathrm{NH}_{2}\right), 3174$ (ArH), 1701 (C=0), 1678 (C=0); ${ }^{1} \mathrm{H}$ NMR (500 MHz, DMSO-d6) 
$\delta: 1.01\left(\mathrm{t}, 3 \mathrm{H}, J=6.8 \mathrm{~Hz}, \mathrm{CH}_{3}\right), 2.12\left(\mathrm{~s}, 3 \mathrm{H}, \mathrm{CH}_{3}\right), 3.88(\mathrm{q}, 2 \mathrm{H}, J=$ $\left.6.8 \mathrm{~Hz}, \mathrm{CH}_{2}\right), 4.33(\mathrm{~s}, 1 \mathrm{H}, \mathrm{CH}), 6.32(\mathrm{~s}, 1 \mathrm{H}, \mathrm{C}=\mathrm{CH}), 6.86(\mathrm{~s}, 2 \mathrm{H}$, $\mathrm{NH}_{2}$ ), 7.22 (d, 2H, $\left.J=8.4 \mathrm{~Hz}, \mathrm{Ar}-\mathrm{H}\right), 7.34(\mathrm{~d}, 2 \mathrm{H}, J=8.4 \mathrm{~Hz}, \mathrm{Ar}-\mathrm{H})$ ppm; ${ }^{13} \mathrm{C}$ NMR $\left(125 \mathrm{MHz}\right.$, DMSO- $\left.d_{6}\right) \delta: 13.9,23.2,26.6,57.3$, 59.0, 101.3, 102.7, 125.8, 128.7, 129.6, 137.7, 144.3, 161.4, 164.7, 167.3, 171.2 ppm; MS(ESI): $M r / z 346$ [M+H]+; Anal. Calcd for $\mathrm{C}_{18} \mathrm{H}_{16} \mathrm{FNO}_{5}$ : C $62.61 \%$, $\mathrm{H} 4.64 \%$, N 4.06\%; Found: $\mathrm{C}$ $62.55 \%$, H $4.60 \%$, N 4.03\%.

Ethyl 2-amino-7-methyl-5-oxo-4-(4-bromophenyl)-4H,5Hpyrano[4,3-b]pyran-3-carboxylate (4I). Pale yellow crystals; m.p.: $194-196{ }^{\circ} \mathrm{C}$; IR (KBr, cm$\left.{ }^{-1}\right): 3381$ and $3316\left(\mathrm{NH}_{2}\right), 3169$ $(\mathrm{ArH}), 1688(\mathrm{C}=0), 1673(\mathrm{C}=0)$; ${ }^{1} \mathrm{H}$ NMR (500 MHz, DMSO-d 6 ) $\delta: 0.97\left(\mathrm{t}, 3 \mathrm{H}, J=6.8 \mathrm{~Hz}, \mathrm{CH}_{3}\right), 2.08\left(\mathrm{~s}, 3 \mathrm{H}, \mathrm{CH}_{3}\right), 3.97(\mathrm{q}, 2 \mathrm{H}, J=$ $\left.6.8 \mathrm{~Hz}, \mathrm{CH}_{2}\right), 4.42(\mathrm{~s}, 1 \mathrm{H}, \mathrm{CH}), 6.35(\mathrm{~s}, 1 \mathrm{H}, \mathrm{C}=\mathrm{CH}), 6.75(\mathrm{~s}, 2 \mathrm{H}$, $\mathrm{NH}_{2}$ ), 7.14 (d, 2H, $\left.J=8.4 \mathrm{~Hz}, \mathrm{Ar}-\mathrm{H}\right), 7.39$ (d, 2H, $J=8.4 \mathrm{~Hz}, \mathrm{Ar}-\mathrm{H}$ ) ppm; ${ }^{13} \mathrm{C}$ NMR (125 MHz, DMSO- $\left.d_{6}\right) \delta: 14.2,22.9,27.2,56.9$, 59.7, 101.0, 103.1, 126.3, 129.0, 129.9, 138.0, 143.7, 161.0, 165.4, 166.4, 171.8 ppm; MS(ESI): $M r / z 406.5$ [M+H]+; Anal. Calcd for $\mathrm{C}_{18} \mathrm{H}_{16} \mathrm{BrNO}_{5}$ : C 53.22\%, H 3.94\%, N 3.45\%; Found: C 53.11\%, H 3.92\%, N 3.44\%.

Ethyl 2-amino-7-methyl-5-oxo-4-(2,4-dichlorophenyl)-4H, $5 H$-pyrano[4,3- $b$ ]pyran-3-carboxylate $(\mathbf{4 m})$. Pale yellow crystals; m.p.: 206-208 ${ }^{\circ} \mathrm{C}$; IR (KBr, cm$\left.{ }^{-1}\right)$ : 3374 and $3304\left(\mathrm{NH}_{2}\right)$, 3182 (ArH), $1695(\mathrm{C}=0), 1680(\mathrm{C}=0)$; ${ }^{1} \mathrm{H}$ NMR (500 MHz, DMSO- $\left.d_{6}\right) \delta: 1.03\left(\mathrm{t}, 3 \mathrm{H}, J=6.8 \mathrm{~Hz}, \mathrm{CH}_{3}\right), 2.11\left(\mathrm{~s}, 3 \mathrm{H}, \mathrm{CH}_{3}\right), 3.93$ (q, $\left.2 \mathrm{H}, J=6.8 \mathrm{~Hz}, \mathrm{CH}_{2}\right), 4.47(\mathrm{~s}, 1 \mathrm{H}, \mathrm{CH}), 6.41(\mathrm{~s}, 1 \mathrm{H}, \mathrm{C}=\mathrm{CH}), 6.83$ (s, $2 \mathrm{H}, \mathrm{NH}_{2}$ ), $7.16(\mathrm{~d}, 1 \mathrm{H}, J=8.4 \mathrm{~Hz}, \mathrm{Ar}-\mathrm{H}), 7.33$ (dd, $1 \mathrm{H}, J=8.4$ $\mathrm{Hz}, J^{\prime}=2.4 \mathrm{~Hz}, \mathrm{Ar}-\mathrm{H}$ ), 7.49 (d, 1H, $J=2.4 \mathrm{~Hz}, \mathrm{Ar}-\mathrm{H}$ ) ppm; ${ }^{13} \mathrm{C}$ NMR (125 MHz, DMSO- $\left.d_{6}\right) \delta: 13.6,23.4,27.0,57.1,59.3,100.8$, 103.4, 126.1, 128.6, 129.7, 137.7, 144.6, 160.5, 164.8, 167.7, $172.3 \mathrm{ppm}$; MS(ESI): $M \mathrm{r} / z 396.5[\mathrm{M}+\mathrm{H}]^{+}$; Anal. Calcd for $\mathrm{C}_{18} \mathrm{H}_{15} \mathrm{Cl}_{2} \mathrm{NO}_{5}$ : C 54.56\%, H 3.79\%, N 3.54\%; Found: C 54.50\%, H $3.77 \%$, N 3.56\%.

Ethyl 2-amino-7-methyl-5-oxo-4-(3,4-dichlorophenyl)-4H, $5 H$-pyrano[4,3-b]pyran-3-carboxylate (4n). Pale yellow crystals; m.p.: $180-181{ }^{\circ} \mathrm{C}$; IR (KBr, cm-1): 3385 and $3309\left(\mathrm{NH}_{2}\right)$, $3176(\mathrm{ArH}), 1707(\mathrm{C}=0), 1673(\mathrm{C}=0)$ ) ${ }^{1} \mathrm{H}$ NMR (500 MHz, DMSO- $\left.d_{6}\right) \delta: 0.98\left(\mathrm{t}, 3 \mathrm{H}, J=6.8 \mathrm{~Hz}, \mathrm{CH}_{3}\right), 2.20\left(\mathrm{~s}, 3 \mathrm{H}, \mathrm{CH}_{3}\right), 3.89$ $\left(\mathrm{q}, 2 \mathrm{H}, J=6.8 \mathrm{~Hz}, \mathrm{CH}_{2}\right), 4.44(\mathrm{~s}, 1 \mathrm{H}, \mathrm{CH}), 6.36(\mathrm{~s}, 1 \mathrm{H}, \mathrm{C}=\mathrm{CH}), 6.77$ (s, $2 \mathrm{H}, \mathrm{NH}_{2}$ ), 7.22 (dd, $1 \mathrm{H}, J=8.8 \mathrm{~Hz}, J^{\prime}=2.0 \mathrm{~Hz}, \mathrm{Ar}-\mathrm{H}$ ), 7.34 (d, $1 \mathrm{H}, J=2.0 \mathrm{~Hz}, \mathrm{Ar}-\mathrm{H}), 7.59$ (d, $1 \mathrm{H}, J=8.8 \mathrm{~Hz}, \mathrm{Ar}-\mathrm{H}) \mathrm{ppm} ;{ }^{13} \mathrm{C}$ NMR (125 MHz, DMSO-d6) $\delta: 14.0,22.7,26.8,56.7,59.5,100.6,102.9$, 125.6, 129.3, 130.7, 138.2, 144.7, 160.9, 165.1, 166.9, 172.5 ppm; MS(ESI): $\quad M r / z \quad 396.8 \quad[\mathrm{M}+\mathrm{H}]+$; Anal. Calcd for $\mathrm{C}_{18} \mathrm{H}_{15} \mathrm{Cl}_{2} \mathrm{NO}_{5}$ : C 54.56\%, H 3.79\%, N 3.54\%; Found: C 54.44\%, H 3.79\%, N 3.50\%.

Ethyl 2-amino-7-methyl-5-oxo-4-(4-methylphenyl)-4H,5Hpyrano[4,3-b]pyran-3-carboxylate (4o). Pale yellow crystals; m.p.: $200-202^{\circ} \mathrm{C}$; IR $\left(\mathrm{KBr}, \mathrm{cm}^{-1}\right): 3370$ and $3314\left(\mathrm{NH}_{2}\right), 3170$ (ArH), 1708 (C=0), 1677 (C=0); ${ }^{1} \mathrm{H}$ NMR (500 MHz, DMSO-d $)_{6}$ $\delta: 1.03\left(\mathrm{t}, 3 \mathrm{H}, J=6.8 \mathrm{~Hz}, \mathrm{CH}_{3}\right), 2.14\left(\mathrm{~s}, 3 \mathrm{H}, \mathrm{CH}_{3}\right), 2.19(\mathrm{~s}, 3 \mathrm{H}$, $\mathrm{CH}_{3}$ ), $3.84\left(\mathrm{q}, 2 \mathrm{H}, J=7.0 \mathrm{~Hz}, \mathrm{CH}_{2}\right), 4.39(\mathrm{~s}, 1 \mathrm{H}, \mathrm{CH}), 6.37$ (s, 1H, $\mathrm{C}=\mathrm{CH}$ ), 6.88 (s, 2H, NH $\mathrm{N}_{2}$ ), 7.19 (d, 2H, J = 8.2 Hz, Ar-H), 7.30 (d, $2 \mathrm{H}, J=8.2 \mathrm{~Hz}, \mathrm{Ar}-\mathrm{H}) \mathrm{ppm} ;{ }^{13} \mathrm{C}$ NMR (125 MHz, DMSO-d6) $\delta: 14.1$, 20.1, 23.3, 26.8, 57.0, 59.3, 101.5, 102.8, 125.5, 128.6, 129.7, 138.0, 144.1, 161.6, 164.4, 167.2, $171.5 \mathrm{ppm}$; MS(ESI): $M r / z$ $342[\mathrm{M}+\mathrm{H}]^{+}$; Anal. Calcd for $\mathrm{C}_{19} \mathrm{H}_{19} \mathrm{NO}_{5}$ : C $66.86 \%, \mathrm{H}$ 5.57\%, N
4.11\%; Found: C 66.80\%, H 5.54\%, N 4.07\%.

Ethyl 2-amino-7-methyl-5-oxo-4-(4-methoxyphenyl)-4H, $5 H$-pyrano[4,3- $b$ ]pyran-3-carboxylate (4p). Pale yellow crystals; m.p.: $212-214{ }^{\circ} \mathrm{C}$; IR ( $\left.\mathrm{KBr}, \mathrm{cm}^{-1}\right): 3367$ and $3307\left(\mathrm{NH}_{2}\right)$, 3178 (ArH), 1712 (C=0), 1683 (C=0); ${ }^{1} \mathrm{H}$ NMR (500 MHz, DMSO-d $d_{6} \delta: 1.07\left(\mathrm{t}, 3 \mathrm{H}, J=6.8 \mathrm{~Hz}, \mathrm{CH}_{3}\right), 2.13\left(\mathrm{~s}, 3 \mathrm{H}, \mathrm{CH}_{3}\right), 3.60$ (s, $\left.3 \mathrm{H}, \mathrm{OCH}_{3}\right), 3.89$ (q, 2H, J = 7.0 Hz, CH 2$), 4.36(\mathrm{~s}, 1 \mathrm{H}, \mathrm{CH}), 6.34$ (s, 1H, C=CH), 6.89 (s, 2H, NH 2$), 7.26$ (d, 2H, $J=8.3 \mathrm{~Hz}, \mathrm{Ar}-\mathrm{H}$ ), 7.40 (d, 2H, $J=8.3 \mathrm{~Hz}$, Ar-H) ppm; ${ }^{13} \mathrm{C}$ NMR $(125 \mathrm{MHz}$, DMSO- $\left.d_{6}\right) \delta$ : 13.7, 22.9, 26.5, 54.2, 57.1, 59.1, 101.4, 103.1, 125.7, 128.5, 129.5, 137.6, 144.7, 162.0, 164.4, 167.4, 171.1 ppm; MS(ESI): $M r / z 358$ [M+H] ${ }^{+}$; Anal. Calcd for $\mathrm{C}_{19} \mathrm{H}_{19} \mathrm{NO}_{6}: \mathrm{C}$ $63.86 \%$, H 5.32\%, N 3.92\%; Found: C 63.78\%, H 5.27\%, N $3.89 \%$.

\section{Results and discussion}

The main objective of the current study was to develop an efficient and simple method for the synthesis of pyrano[4,3-b]pyran derivatives in a non-toxic solvent. The reaction of 4-bromobenzaldehyde (1a) with malononitrile (2a) and 4-hydroxy-6-methylpyran-2-one (3) to afford 2-amino-5oxo-7-methyl-4-(4-bromophenyl)- $4 H, 5 H$-pyrano[4,3-b]pyran-3 -carbonitrile (4a) was used as a model reaction to identify suitable conditions for the transformation using TUD as a catalyst (Scheme 1).

To optimize the reaction conditions and evaluate the catalytic activity of TUD compared with other catalysts for the formation of $\mathbf{4 a}$, comparative experiments were performed and the data are shown in Table 1. Among the different catalysts tested, including $\mathrm{LiBr}, \mathrm{NH}_{4} \mathrm{Cl}$, sulfamic acid, $p$-TSA, $\mathrm{HBF}_{4}$ and TUD, TUD was found to be the most efficient in terms of the reaction time and yield of the product (Table 1, Entry 1-6). We then proceeded to investigate the effect of different catalyst loadings on this transformation in water at $80{ }^{\circ} \mathrm{C}$ (Table 1 , Entry $6-10$ ). Catalyst loadings in the range of $0-15 \%$ were tested. A low yield of the product was observed in the absence of the catalyst, demonstrating that the catalyst is essential for formation of the product in high yield. The use of lower amounts of the catalyst, such as $2 \%$ and $5 \%$, afforded poorer yields of

\section{Table 1}

Evaluation of the catalytic activity of different catalysts for the condensation of 4-bromobenzaldehyde with malononitrile and 4-hydroxy-5methylpyran-2-one in water at $80^{\circ} \mathrm{C}$.

\begin{tabular}{lcccc}
\hline Entry & Catalyst & Loading $(x / \%)$ & Time $(\mathrm{min})$ & Yield $^{\mathrm{a}}(\%)$ \\
\hline 1 & $\mathrm{LiBr}$ & 10 & 120 & 36 \\
2 & $\mathrm{NH}_{4} \mathrm{Cl}$ & 10 & 300 & 44 \\
3 & Sulfamic acid & 10 & 300 & 39 \\
4 & $p$-TSA & 10 & 300 & 47 \\
5 & $\mathrm{HBF}_{4}$ & 10 & 300 & 51 \\
6 & $\mathrm{TUD}$ & 10 & 40 & 92 \\
7 & TUD & 0 & 300 & 34 \\
8 & TUD & 2 & 60 & 63 \\
9 & TUD & 5 & 50 & 78 \\
10 & TUD & 15 & 40 & 93 \\
\hline
\end{tabular}

Reaction conditions: 4-Bromobenzaldehyde (1.0 mmol), malononitrile (1.2 mmol) and 4-Hydroxy-6-methylpyran-2-one (1.0 mmol). asolated yield. 
Table 2

Synthesis of 2-amino-7-methyl-5-oxo-4-(4-bromophenyl)-4H,5 $H$-pyrano $[4,3-b]$ pyran-3-carbonitrile in the presence of the TUD catalyst $(x=$ $10 \%)$ under different reaction conditions.

\begin{tabular}{lcccc}
\hline Entry & Solvent & Temperature $\left({ }^{\circ} \mathrm{C}\right)$ & Time $(\mathrm{min})$ & Yield ${ }^{\mathrm{a}}(\%)$ \\
\hline 1 & 1,4-Dioxane & Reflux & 90 & 51 \\
2 & $\mathrm{CH}_{3} \mathrm{CN}$ & Reflux & 90 & 55 \\
3 & $\mathrm{THF}$ & Reflux & 90 & 48 \\
4 & $\mathrm{CH}_{2} \mathrm{Cl}_{2}$ & Reflux & 90 & 43 \\
5 & $\mathrm{CHCl}_{3}$ & Reflux & 90 & 36 \\
6 & $\mathrm{MeOH}$ & Reflux & 60 & 72 \\
7 & $\mathrm{EtOH}$ & Reflux & 60 & 74 \\
8 & $\mathrm{H}_{2} \mathrm{O}$ & 80 & 40 & 92 \\
9 & $\mathrm{H}_{2} \mathrm{O}$ & rt & 120 & 48 \\
10 & $\mathrm{H}_{2} \mathrm{O}$ & 50 & 90 & 62 \\
11 & $\mathrm{H}_{2} \mathrm{O}$ & 60 & 60 & 72 \\
12 & $\mathrm{H}_{2} \mathrm{O}$ & 70 & 50 & 81 \\
13 & $\mathrm{H}_{2} \mathrm{O}$ & 90 & 40 & 93 \\
\hline
\end{tabular}

Reaction conditions: 4-bromobenzaldehyde $(1 \mathrm{mmol})$, malononitrile (1.2 mmol) and 4-hydroxy-6-methylpyran-2-one (1 mmol).

asolated yield.

the desired product. Furthermore, an increase in the amount of the catalyst to $15 \%$ did not lead to a significant improvement in the results, demonstrating that a catalyst loading of 10\% TUD in water was sufficient to push this reaction forward, and the results are summarized in Table 1.

To evaluate the effects of different solvents on the reaction, the model reaction was conducted in a range of different solvents, including 1,4-dioxane, $\mathrm{CH}_{3} \mathrm{CN}$, THF, $\mathrm{CH}_{2} \mathrm{Cl}_{2}, \mathrm{CHCl}_{3}, \mathrm{MeOH}$, $\mathrm{EtOH}$ and water under reflux conditions in the presence of $10 \%$
TUD catalyst (Table 2, Entry 1-8). Aprotic solvents gave the desired product in moderate yields, whereas protic solvents gave the product in good to excellent yields. Water was clearly identified as the solvent of choice because it provided the highest yield of the desired product, as well as being the most cost-effective and environmental acceptability of the solvents tested (Table 2, Entry 8).

Having optimized the solvent for the reaction, we proceeded to investigate the use of different temperatures including room temperature and 50, 60, 70, 80 and $90^{\circ} \mathrm{C}$ (Table 2, Entry 8-13). Increasing the temperature of the reaction from room temperature to $80{ }^{\circ} \mathrm{C}$ led to a reduction in the reaction time. The greatest yield in the shortest reaction time was obtained in water at $80{ }^{\circ} \mathrm{C}$ (Table 2, Entry 8). The use of water as the reaction media was not only advantageous from an economical perspective but was also beneficial in terms of the environmental and green chemistry characteristics of the process.

To evaluate the scope of this catalytic transformation, the optimized reaction conditions were subsequently applied to the reaction of malononitrile or cyanoacetate and 4-hydroxy6-methylpyran-2-one with a variety of different aromatic aldehydes (Table 3, Entry 1-16). A wide range of aromatic aldehydes bearing either electron-donating or electron-withdrawing substituents reacted successfully with malononitrile or cyanoacetate and 4-hydroxy-6-methylpyran -2-one to give the corresponding pyrano[4,3- $b]$ pyran derivatives in high yields over short reaction time. In all cases, the reaction was found to be selective and afforded good to high yields of the desired products without any evidence of the formation of any side products.

Having successfully demonstrated the scope of the opti-

Table 3

Synthesis of various pyrano[4,3-b]pyran-3-carbonitrile derivatives.

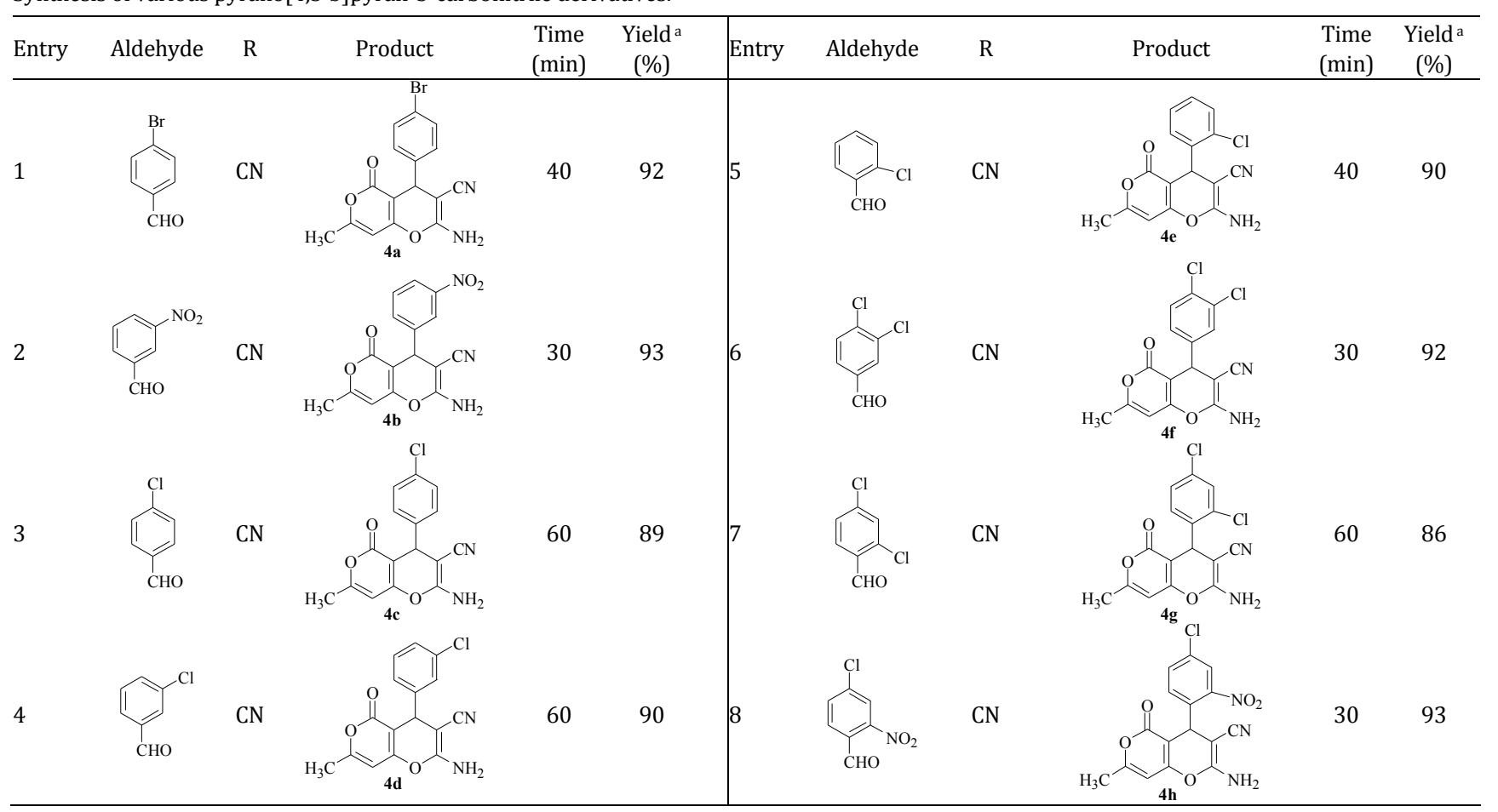


Table 3 (continued)

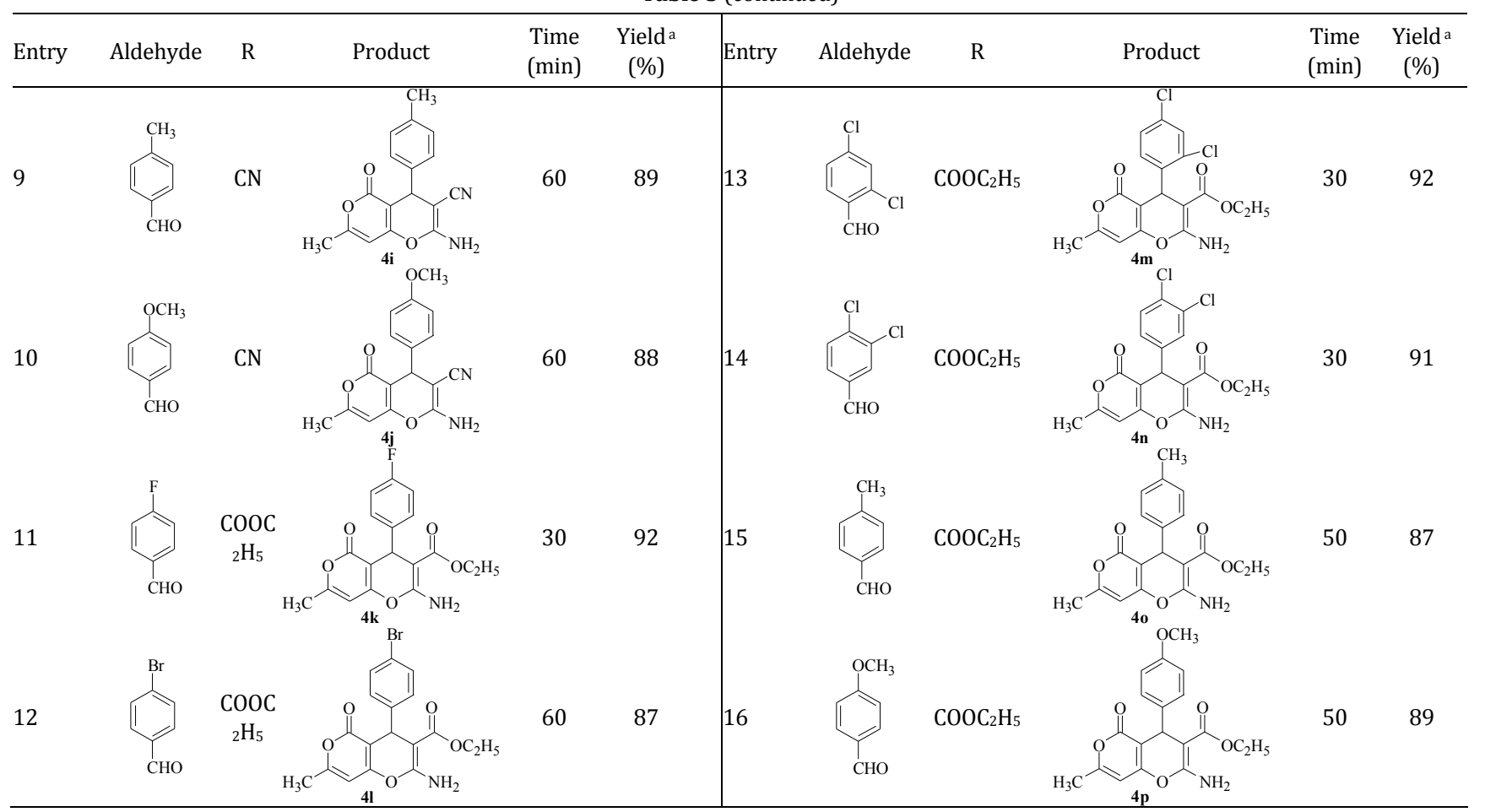

Reaction conditions: Aromatic aldehydes (1.0 mmol), malononitrile or cyanoester (1.2 mmol) and 4-hydroxy-6-methylpyran-2-one (1.0 mmol) in water at $80{ }^{\circ} \mathrm{C}$ in the presence of TUD $(x=10 \%)$.

asolated yields.

mized reaction conditions, we proceeded to evaluate the recyclability of the catalyst by performing the reaction of 4-bromobenzaldehyde, malononitrile and 4-hydroxy-5methylpyran-2-one in the presence of catalytic amount of TUD $(x=10 \%)$ in water. Upon completion of the reaction, the mixture was poured into water $(100 \mathrm{ml})$, and the resulting solid was removed by filtration and washed with water. The remaining aqueous solution of TUD was reused without any further treatment for the subsequent run. The recyclability of the aqueous TUD was tested in this way over five runs, with the desired product being isolated in similar yields over similar reaction time for each run. The results of these recycling experiments are summarized in Table 4. These results demonstrated that the catalyst could be efficiently recycled to produce consistent levels of activity.

The FT-IR spectrum for $\mathbf{4 a}$ exhibited sharp bands at 3377 and $3307\left(\mathrm{NH}_{2}\right), 2222(\mathrm{CN})$, and $1706 \mathrm{~cm}^{-1}(\mathrm{C}=0)$. The ${ }^{1} \mathrm{H}$ NMR

Table 4

Effect of recycling the TUD catalyst on the yield of 2-amino-7-methyl -5-oxo-4-(4-bromophenyl)-4H,5H-pyrano[4,3-b]pyran-3-carbonitrile.

\begin{tabular}{lccc}
\hline Entry & Cycle & Time $(\mathrm{min})$ & Yield $^{\mathrm{a}}(\%)$ \\
\hline 1 & 0 & 40 & 92 \\
2 & 1 & 40 & 91 \\
3 & 2 & 40 & 89 \\
4 & 3 & 40 & 87 \\
5 & 4 & 40 & 87 \\
\hline
\end{tabular}

Reaction conditions: 4-Bromobenzaldehyde (1.0 mmol), malononitrile $(1.2 \mathrm{mmol})$ and 4-hydroxy-6-methylpyran-2-one $(1.0 \mathrm{mmol})$ in water at $80^{\circ} \mathrm{C}$ in the presence of TUD $(x=10 \%)$.

a Isolated yields. spectrum of $\mathbf{4 a}$ contained a singlet peak, which was identified as the resonance signal of a methyl group (2.23 ppm), as well as two other singlets at 4.42 and $6.52 \mathrm{ppm}$, which were identified as two methines (i.e., $\mathrm{CH}$ and $\mathrm{CH}=$ ), respectively. One other singlet peak was observed at $6.77 \mathrm{ppm}$, which was identified as the $\mathrm{NH}_{2}$ resonance signal, as well as a series of multiplets (7.17-7.44 ppm) corresponding to the aromatic protons.

Although the exact mechanism of the current reaction is not known at this stage, we have proposed a mechanism (Scheme 2) based on existing reports in the literature [18]. It was assumed that TUD is an effective catalyst for the formation of the olefin 5, which would be formed in situ by the Knoevenagel condensation of aryl aldehyde $\mathbf{1}$ and the active methylene compound 2. Olefin 5 could then react with 4-hydroxy-5methylpyran-2-one to give intermediate $\mathbf{6}$. The subsequent cyclization of 6, as shown in Scheme 2, would afford intermediate 7 , which would tautomerize to yield corresponding pyrano[4,3-b]pyran derivative 4 .

\section{Conclusions}

In conclusion, we have developed a simple and highly efficient one-pot three-component method for the synthesis of a range of pyrano[4,3- $b$ ]pyran-5-one derivatives catalyzed by TUD. The TUD catalyst was easily prepared by the oxidation of thiourea with hydrogen peroxide. In general, the reactions of different substrates were fast, clean and, in most cases, required no chromatographic purification, with the desired products being isolated in excellent yields. There are several attractive features to this procedure, including its operational 


\section{Graphical Abstract}

Chin. J. Catal., 2014, 35: 127-133 doi: 10.1016/S1872-2067(12)60727-X

Thiourea dioxide: An efficient and reusable organocatalyst for the rapid one-pot synthesis of pyrano[4,3-b]pyran derivatives in water

Majid Ghashang, Syed Sheik Mansoor*, Krishnamoorthy Aswin Islamic Azad University, Iran;

C. Abdul Hakeem College, India

A series of pyrano[4,3- $b]$ pyran derivatives have been synthesized by the reaction of aromatic aldehydes with malononitrile or cyanoacetate and 4-hydroxy-6-methylpyran-2-one in water at $80^{\circ} \mathrm{C}$ using an aqueous solution of thiourea dioxide as a catalyst in excellent yields.

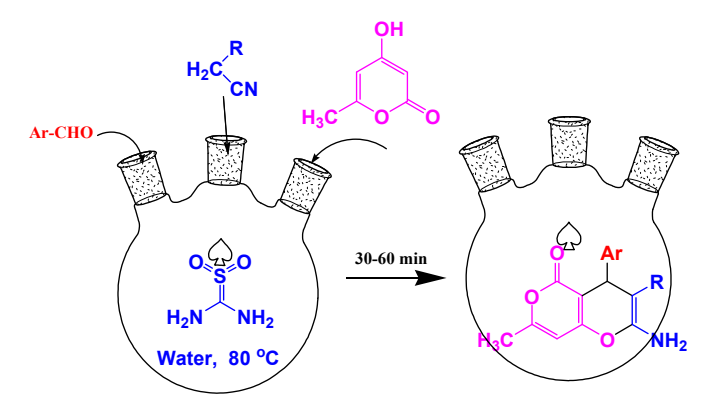

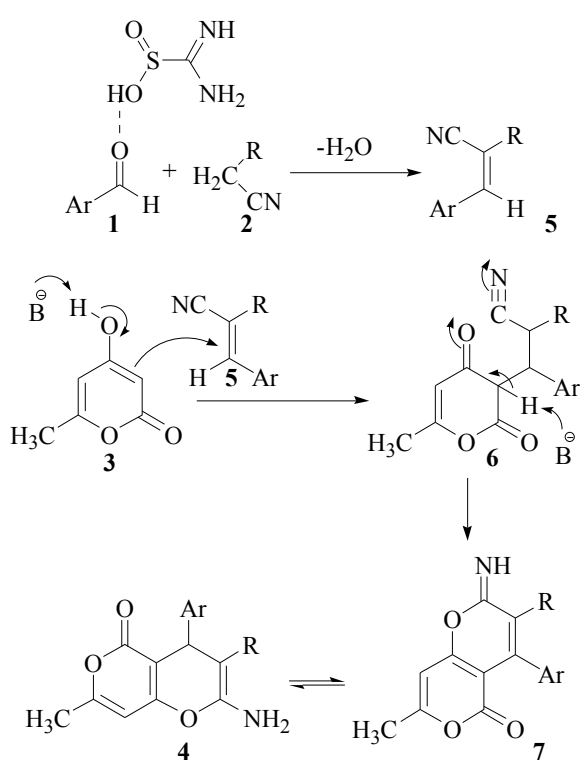

Scheme 2. A possible mechanism for the formation of the pyrano[4,3-b]pyran derivatives.

simplicity, high yield, and facile work-up and purification. Furthermore, the use of TUD as an inexpensive and non-volatile catalyst makes the method environmentally friendly and economically viable.

\section{Acknowledgments}

The author Mansoor expresses his gratitude to the management of C. Abdul Hakeem College, Melvisharam - India, for the facilities and support.

\section{References}

[1] Domling A, Ugi I. Angew Chem, Int Ed, 2000, 39: 3168

[2] Heck S, Domling A. Synlett, 2000: 424

[3] Davoodnia A, Khashi M, Tavakoli-Hoseini N. Chin J Catal (催化学 报), 2013, 34: 1173

[4] Azizi N, Davoudpour A, Eskandari F, Batebi E. Monatsh Chem, 2013, 144: 405
[5] Martinez-Grau A, Marco J L. Bioorg Med Chem Lett, 1997, 7: 3165

[6] Hiramoto K, Nasuhara A, Michikoshi K, Kato T, Kikugawa K. Mutat Res, 1997, 395: 47

[7] Makawana J A, Patel M P, Patel R G. Arch Pharm (Weinheim). 2012, 345: 314

[8] Venkatesham A, Rao R S, Nagaiah K, Yadav J S, Roopa-Jones G, Basha S J, Sridhar B, Addlagatta A. Med Chem Comm, 2012, 3: 652

[9] Bianchi G, Tava A. Agric Biol Chem, 1987, 51: 2001

[10] Mohr S J, Chirigos M A, Fuhrman F S, Pryor J W. Cancer Res, 1975, 35: 3750

[11] Kamdar N R, Haveliwala D D, Mistry P T, Patel S K. Eur J Med Chem, 2010, 45: 5056

[12] Hazeri N, Maghsoodlou M T, Mousavi M R, Aboonajmi J, Safarzaei M. Res Chem Intermed, 2013, doi: 10.1007/s11164-013-1179-z

[13] Nasseri M A, Sadeghzadeh S M. Monatsh Chem, 2013,144: 1551

[14] Khurana J M, Kumar S. Tetrahedron Lett, 2009, 50: 4125

[15] Pratap U R, Jawale D V, Netankar P D, Mane R A. Tetrahedron Lett, 2011, 52: 5817

[16] Salvi P P, Mandhare A M, Sartape A S, Pawar D K, Han S H, Kolekar S S. C R Chim, 2011, 14: 878

[17] Hasaninejad A, Shekouhy M, Golzar N, Zare A, Doroodmand M M. Appl Catal A, 2011, 402: 11

[18] Wang X S, Zeng Z S, Li Y L, Shi D Q Tu S J, Zhou J X. ARKIVOC, 2006: 107

[19] Rajguru D, Keshwal B S, Jain S, Bhagwat V W. Monatsh Chem, 2013, doi: 10.1007/s00706-013-0975-x

[20] Mirza-Aghayan M, Saravani F, Tarlani A A, Abaee M S, Boukherroub R. Monatsh Chem, 2013, doi: 10.1007/s00706-0131035-2

[21] Rajguru D, Keshwal B S, Jain S. Med Chem Res, 2013, doi: 10.1007/ s00044-013-0586-4

[22] Verma S, Kumar S, Jain S L, Sain B. Org Biomol Chem, 2011, 9: 6943

[23] Kumar S, Jain S L, Sain B. RSC Advances, 2012, 2: 789

[24] Verma S, Jain S L. Tetrahedron Lett, 2012, 53: 6055

[25] Ohura O, Fujimoto O. US Patent 4233 238. 1980

[26] Ghashang M, Mansoor S S, Aswin K. J Adv Res, 2013, doi: 10.1016/j.jare.2013.03.003

[27] Ghashang M, Aswin K, Mansoor S S. Res Chem Intermed, 2013, doi: 10.1007/s11164-013-1027-1

[28] Mansoor S S, Aswin K, Logaiya K, Sudhan P N, Malik S. Res Chem Intermed, 2012, doi: 10.1007/s11164-012-1008-9

[29] Ghashang M, Mansoor S S, Aswin K. Res Chem Intermed, 2013, doi: 10.1007/s11164-013-1419-2 